Maria Cardillo

La Sapienza, Università di Roma

Dipartimento di Lettere e Culture Moderne

https://doi.org/10.18778/8220-478-0.18

\title{
ANTONIO CANOVA E IL RITRATTO DI HENRYK LUBOMIRSKI TRA SCRITTURE PRIVATE E PUBBLICISTICA D’ARTE
}

\begin{abstract}
Il contributo intende far luce sulla genesi del ritratto scultoreo di Henryk Lubomirski come Amore realizzato da Antonio Canova su commissione di Elżbieta Czartoryska Lubomirska nella primavera del 1786 a Roma. Molti dettagli si apprendono dalle fonti documentarie quali le annotazioni private dell'artista edite con il titolo di Scritti, nei quali l'artista raccontava in modo conciso la sua attività artistica, dalle biografie coeve o di poco posteriori e dal periodo romano "Memorie per le belle arti” (1785-1788).
\end{abstract}

Parole chiave: A. Canova, H. Lubomirski, scultura, neoclassicismo, Settecento, fonti

Abstract: This contribution aims to clear up the circumstances that allowed Antonio Canova to realize the sculptural portrait of Prince Henryk Lubomirski in guise of Cupid, commissionated by Princess Elżbieta Czartoryska Lubomirska during the spring of 1786 in Rome. Many details are known through documentary sources like Canova's Writings (Scritti) in which soberly recounted his artistic activity, nearcontemporary biographies and eventually a roman periodical paper dedicated to arts called "Memorie per le belle arti" (1785-1788).

Keywords: A. Canova, H. Lubomirski, sculture, Neoclassicism, $18^{\text {th }}$ century, sources

All'opposito de' moderni artefici i quali si credono di dar maggior saggio del saper loro, quanto più ne fanno spiccar le vene ed i muscoli nelle immagini di qualsivoglia età, appresso i Greci le figure della pubertà $[\ldots]$ eran quelle ove ponevasi tutto il sapere e lo studio, per far risaltare la bellezza, ed ove il genio e la delicatezza del senso loro dava saggio di sé, avendo scelto piuttosto 
questa che ogni altra età, e talor a dispetto della mitologia; essendosi avveduti ben eglino [i Greci], che la gioventù sola era atta a rappresentarne le divinità rivestite di corpi sí, ma aerei anziché materiali ${ }^{1}$.

Così sosteneva Winckelmann nel breve trattato Dell'arte del disegno de' Greci e della bellezza preliminare al quarto capitolo del primo tomo de I monumenti antichi inediti che fu scritto in lingua italiana ed edito in prima edizione a Roma nel 1767 dai Pagliarini ${ }^{2}$, punta avanzata dell'editoria artistica romana di fine del Settecento da cui torchi uscirono alcune pietre miliari dell'estetica neoclassica, come gli scritti del «pittor filosofo» ossia Anton Raphael Mengs (1728-1779) e quelli di Carlo Fea (1753-1836), solo per fare qualche nome di spicco.

Secondo lo studioso tedesco la massima espressione della bellezza si trovava nelle «figure della pubertà»e, sicuramente, tali parole non furono estranee ad Antonio Canova (1757-1822) nell'atto di scolpire il ritratto del giovane Henryk Lubomirski (1777-1850) oggetto di questo contributo, intendendo quindi declinare il concetto di italianità come estro, creatività nonché gusto naturalmente incline all'armonia e alla bellezza ancora oggi vivo e presente nel cosiddetto made in Italy.

Lambiente artistico romano della seconda metà del XVIII secolo fu ampiamente dominato dalla figura di Antonio Canova. All'età di 22 anni egli si trasferiva a Roma al fine di perfezionare gli studi artistici. Nell'Urbe lo scultore stringeva amicizia con tre illustri personaggi destinati a influenzare il suo percorso: Giovanni Volpato ${ }^{3}$ (1735-1803), Gavin Hamilton ${ }^{4}$ (1723-1798) e l'ambasciatore veneto e noto collezionista Girolamo Zulian (1730-1795). L'incisore Giovanni Volpato, suo conterraneo nativo di Bassano del Grappa (Vicenza), rappresentava un punto di riferimento per la comunità di artisti veneti operanti a Roma. Fu lui, infatti, a procurare a Canova il suo primo importante lavoro ovvero il monumento funerario a papa a Clemente XIV nella basilica dei Santi Apostoli. Lamicizia con Hamilton si rivelava altrettanto determinante, ma più sotto il profilo artistico, perché il pittore scozzese contribuì ad arricchire il gusto estetico del Canova, comunicandogli un raffinato ed elegante «culto della

1 J. J. Winckelmann (1767), Dell'arte del disegno de' Greci e della bellezza, in Monumenti antichi inediti, vol. 1, Roma, [Pagliarini], 2 voll, p. 36.

2 S. Franchi (1994-2002), Le impressioni sceniche. Dizionario bio-bibliografico degli editori e stampatori romani e laziali di testi drammatici e libretti musicali dal 1579 al 1800, Roma, Edizioni di storia e letteratura, pp. 582-586.

3 G. Marini (a c. di) (1988), Volpato Giovanni 1735-1803, Bassano del Grappa (Vicenza), Ghedina \& Tassotti.

4 A. Cesareo (2002), "Gavin Hamilton (1723-1798): a gentleman of probity, knowledge and real taste", Saggi e memorie di storia dell'arte 26, pp. 211-322. 
malinconia», ${ }^{5}$ tipico della sensibilità inglese. Fu inoltre tramite Hamilton che Canova conobbe Girolamo Zulian, ambasciatore veneto presso la Santa Sede e mecenate di tanti artisti, il quale offrì un sostegno economico anche a Canova, vitto e alloggio a Palazzo Venezia affinché potesse continuare i suoi studi.

La formazione del Canova passò attraverso una rigorosa analisi della statuaria antica, cominciando dai gessi della Galleria Farsetti a Venezia ${ }^{6}$ per passare quindi ai Musei Vaticani, dove, ottenuto il permesso, il nostro eseguiva copie scrupolose spesso ripetute dello stesso soggetto, come documentano i disegni a matita, realizzati durante i primi mesi del suo soggiorno romano e custoditi oggi al Museo Civico di Bassano ${ }^{7}$. In questi anni, inoltre, lo scultore maturava la convinzione che per raggiungere la piena padronanza dei propri mezzi espressivi gli era necessario approfondire le conoscenze filosofiche e letterarie. In questo senso era soprattutto la mitologia a offrire all'estro dello scultore quelle suggestioni, spunti e immagini che avrebbero alimentato la sua arte. Alla formazione culturale di Canova provvedeva, come abbiamo detto l'ambasciatore Zulian, il quale mise a sua disposizione in qualità di precettore il suo segretario, l'abate Giuseppe Foschi ${ }^{8}$, affinché lo introducesse allo studio e alla lettura dei classici greci e latini e della mitologia.

Sul piano professionale Canova riceveva, come è stato detto, la sua prima importante commissione pubblica agli inizi del 1784, quando ottenne l'incarico di scolpire il momento funebre di papa Clemente XIV. L'opera, destinata a ornare la porta di ingresso della sagrestia della basilica dei Santi Apostoli, venne eretta a spese di Carlo Giorgi (circa 1724-1803), un ricco mercante, che doveva le sue fortune economiche a papa Ganganelli9. Per la realizzazione del monumento Giorgi si rivolse all'incisore veneto Giovanni Volpato, il quale,

5 O. Stefani (1990), I rilievi del Canova. Una nuova concezione del tempo e dello spazio, Milano, Electa, p. 13.

6 A. Munõz (1924/1925), "Il periodo veneziano di Antonio Canova e il suo primo maestro", Bollettino d'arte del Ministero della Pubblica Istruzione 18, pp. 103-128.

7 Si tratta del Album B $(575 \times 434 \mathrm{~mm})$ contenente 43 carte con 56 disegni del primo soggiorno romano compreso tra il 18 ottobre 1779 e 14 giugno 1780. F. Rigon (1982), "Disegni del Canova del Museo di Bassano", [in:] Giulio Carlo Argan, Barbieri Franco, Ferdinando Rigon (a c. di), Disegni del Canova del Museo di Bassano, Milano, Electa: p. 18.

8 Memorie di Antonio Canova scritte da Antonio D'Este e pubblicate per cura di Alessandro D'Este con note e documenti, Firenze, Le Monnier, 1864, pp. 19-20.

9 G. Pavanello (2014), "Su una prima idea di Canova per il monumento funerario di Clemente XIV", [in:] Vincenzo Cazzato, Sebastiano Roberto e Mario Bevilacqua (a c. di) La Festa delle Arti. Scritti in onore di Marcello Fagiolo per i cinquant'anni di studi, Roma, Gangemi, vol. 2, p. 772; A. Zanella (2000), "Il monumento funerario papale da Bernini a Canova", [in:] Giuseppe Pavanello (a c. di) Antonio Canova e il suo ambiente 
scontrandosi con un coro di ostilità, propose il giovane Antonio Canova. Si trattò indubbiamente - come sottolinea Fernando Mazzocca ${ }^{10}$ - di una mossa azzardata, destinata però a cambiare il destino dell'arte scultorea, in quanto segnava la definitiva consacrazione del giovane scultore di Possagno. Questo ai danni di un altro scultore attivo nell'Urbe in quegli anni ossia il romano Giuseppe Angelini ${ }^{11}$ (1738-1811), che aveva ingaggiato una sorta di rivalità con Canova, come lo scultore stesso ricordava nelle sue scritture private, in particolare nell'annotazione del 14 novembre $1780^{12}$. Ma la competizione era impari e per Angelini la sconfitta fu inevitabile, determinando in lui disturbi mentali che lo avrebbero condotto ad abbandonare quasi del tutto lo scalpello. Nel presentare il monumento funebre di papa Clemente XIV la testata romana le "Memorie delle Belle Arti" (1785-1788), cioè una delle prime riviste d'arte in Italia ${ }^{13}$, sottolineava come Canova dimostrasse unattitudine allantico, in particolare ai modelli greci. Del resto, il continuo confrontare Canova a Fidia fa pensare che questa fosse una convinzione diffusa tra i contemporanei ${ }^{14}$.

Mentre Canova stava ancora lavorando al momento funebree, in particolare, alla statua dell'Umiltà e a quella di papa Ganganelli, troviamo scritto nei suoi appunti l'inizio di un nuovo lavoro relativamente a un amorino:

"La settimana de 5 giugno 1786 incominciai il modello in grande dell'Amorino"15

Così è scritto nel Libro dei conti di Canova: un quaderno di 181 fogli vergato di suo pugno, contenente annotazioni di carattere prevalentemente economico del quinquennio romano compreso tra il luglio 1783 e il luglio

artistico fra Venezia, Roma e Parigi, Venezia, Istituto Veneto di Scienze, Lettere ed arti, pp. 277-281.

10 F. Mazzocca (2002), Giovanni Volpato mentore di Canova, [in:] Ferdinando Mazzocca, Enrico Colle (a c. di), Il neoclassicismo in Italia da Tiepolo a Canova, Milano, Skira, p. 369.

11 F. Leone (1999), “Giuseppe Angelini (1738-1811), Storia, committenze e rapporti con l'Europa nel culto della classicità, Ricerche di storia dell'arte 68, p. 9.

12 A. Canova (2007), Scritti, Hugh Honour e Paolo Mariuz (a c. di), Roma, Salerno editrice, p. 65.

13 La rivista è stata oggetto della mia tesi di dottorato (XXVIII ciclo) Belle arti e antichità a Roma al declinare del secolo dei lumi. L'invenzione capitolina del giornalismo artistico italiano discussa nel luglio 2017.

14 F. Mazzocca (2002), Antonio Canova, il "Fidia rinascente" tra antico e classico [in:] Ferdinando Mazzocca, Enrico Colle (a c. di), Il neoclassicismo in Italia da Tiepolo a Canova, Milano, Skira, p. 375.

15 A. Canova, op. cit., p. 222. 
1788. Tale quaderno si trova oggi alla Biblioteca Civica di Bassano per la donazione fatta nel 1851 del fratello dello scultore, mons. Giovanni Battista Sartori (1775-1858).

Inoltre, un'altra nota autografa di Canova vergata nel 1787 e confluita anch'essa alla Biblioteca Civica di Bassano per la donazione del fratello di Canova si legge:

Adesso sto lavorando, o per meglio dire terminando, due Amorini di grandezza all'età di dice anni uno con la testa ritratto di un signorino nepote della prencipessa Lubormirski di Versavia, e l'altro con la testa ideale per il colonello Campbell ${ }^{16}$

Da una biografia anonima, redatta intorno al 1804-1805 e nota come Abbozzo di Biografia, la prima tra le numerose biografie sull'artista ad aggiungere una serie di informazioni inedite sulla via dell'artista, al punto da pensare a qualche forma di revisione da parte dello stesso scultore, leggiamo:

A fronte d'ogni sua ripugnanza per non aver mai fatto alcun ritratto, dovendo cedere finalmente all'importuna insistenza della principessa polacca Lobromiski (sic), si mise a formare in creta il ritratto d'un [nobile] leggiadro di lei nipotino di 12 anni, il signor Enrico Sartoski (sic), ma per la timidezza sua naturale, non poté compierlo se non che da sé solo in assenza del giovinetto. Lo modellò poscia in aprile sotto la figura intiera d'Amore, e lo finì susseguentemente in marmo. Ne sta inserita la descrizione nel tomo IV delle Memorie, che pubblicavansi in Roma dal cavalier De Rossi ${ }^{17}$

Questa preziosa fonte getta una luce sulla genesi del ritratto scultoreo del piccolo principe Henryk Lubomirski ${ }^{18}$ (1777-1850). Innanzitutto, Canova accettava con riluttanza la committenza, perché, oltre agli altri impegni lavorativi, lo scultore non si era ancora cimentato nel ritratto. Secondariamente la timidezza del principe Henryk costringeva Canova a scolpire il volto a memoria, un disagio non da poco per unartista. Eppure, di fronte alle insistenze della principessa Elżbieta Czartoryska Lubomirska (1736-1816), che commissionò il ritratto del nipote adolescente quando quest'ultimo non

16 Ibidem, p. 261.

17 Ibidem, pp. 346-347.

18 Il principe Henryk fu ritratto anche da Elisabeth Vigée-Lebrun (1755-1842), da Maria Cosway (1760-1838) nel 1787 la cui opera andò perduta, da Angelica Kauffmann (1741-1807) nel 1786 e, infine, un ritratto scultoreo fu eseguito nel 1787 da Anne Seymour Damer (1748-1828). 
aveva ancora compito dieci anni nella primavera del $1786^{19}$, Canova non seppe dire di no. Del resto, Elżbieta Lubomirska era una delle dame più in vista in quegli anni a Roma, nota come la «Pompadour di Polonia $»^{20}$. A lei faceva capo una rete di massoneria polacca ed era stata accusata di complottare contro il re Stanislaw II Augusto Poniatowki, salito al trono nel $1764^{21}$.

Dal Libro de' Conti ${ }^{22}$ del Canova si evince che il costo totale lievitò oltre 700 zecchini ma la principessa non badava a spese, avendo grosse disponibilità economiche. Sulle modalità del pagamento Hugh Honour, uno dei massimi studiosi di Canova, aggiunge un dettaglio importante e cioè che la principessa lasciò Roma senza saldare il conto a Canova, il quale si vide costretto a scrivere probabilmente all'abate Scipione Piattoli (1749-1809), precettore di Henryk, per sollecitare il pagamento. Tale lettera andò perduta, ma da un'altra missiva di Piattoli a Canova si apprende che il saldo avvenne tramite il banchiere inglese Thomas Jenkins (circa 1722-1798) ${ }^{23}$.

E, ancora, nel frammento di Abbozzo di Biografia si parla di Giovanni Gherardo De Rossi ${ }^{24}$ (1754-1827) e delle "Memorie delle belle arti" (1784-1788). Fu De Rossi a giocare un ruolo determinante nel promuovere lo scoppiettante ingegno di Canova. Oggi forse questo può sembrare assodato, ma non era così così scontato nel 1787 . È significativo in questo senso che l'anonimo estensore dell'Abbozzo di Biografia abbia commesso lo stesso errore in merito al cognome del piccolo Henryk, Czartoryski invece che Lubomirski, che va inteso come un ulteriore indizio della promozione e autorevolezza del commento di De Rossi. Questi non era quel che si può definire un letterato puro, dal momento che fu banchiere di professione ma letterato per passione, scrisse infatti una raccolta di dialoghi e sedici commedie alla maniera del Goldoni. Ma, ai fini di questa ricerca, De Rossi era soprattutto un collezionista di consolidata esperienza. Si favoleggiava della sua ricca collezione, di cui non possediamo alcun inventario e che andò venduta alla sua morte, e che probabilmente

19 G. Cunial (2007), "Amore che legò per sempre Canova ai Polacchi", [in:] Mario Guderzo (a c. di), Antonio Canova: il principe Henryk Lubomirski come Amore, Cinisello Balsamo (Milano), Silvana editoriale, p. 44.

20 A. Busiri Vici (1988), "I turisti polacchi a Roma nella seconda metà del Settecento", Strenna dei Romanisti 44, pp. 74-75.

21 S. Lorentz (1962), Relazioni artistiche fra l'Italia e la Polonia, Roma, Signorelli.

22 A. Canova, op. cit., p. 246.

23 H. Honour (1973), "Gli Amorini del Canova", Arte illustrata 6, p. 314; H. Honour (2007), Amorini del Canova, [in:] Mario Guderzo (a c. di), Antonio Canova: il principe Henryk Lubomirski come Amore, Cinisello Balsamo (Milano), Silvana editoriale, p. 30.

24 A. Rita (1991), De Rossi, Giovanni Gherardo, [in:] Dizionario Biografico degli Italiani, vol. 39, Roma, Istituto della Enciclopedia Italiana, pp. 214-218. 
conteneva pezzi del Canova. Sicuramente c'era una tela dipinta dal Canova come esercitazione giovanile, che De Rossi volle far credere per scherzo un'originale del Giorgione, come è ricordato da Antonio d'Este ${ }^{25}$ (1754-1837), amico e allievo dello scultore di Possagno, nella biografia di Canova ${ }^{26}$ da lui allestita ed edita nel 1864. De Rossi e Canova si erano conosciuti assai probabilmente tramite Abbondio Rezzonico (1742-1810), anch'egli veneto e committente dello scultore di Possagno, nonché patron delle "Memorie" in cui, oltre a De Rossi scriveva pure Onofrio Boni (1739-1818), poi sostituito da Leonardo Massimiliano De Vegni (1731-1801), anch'egli architetto. Gli articoli della rivista erano dedicati alle cosiddette arti sorelle: pittura, scultura e architettura. I contributi presentavano un'immagine del mercato artistico romano non esente dalla logica della promozione e delle preferenze amicali. Fu De Rossi quindi a dare quella visibilità che l'astro nascente del Canova meritava. La sua fu una fiducia ben risposta, visto che dopo avere recensito il monumento funebre di Clemente XIV, De Rossi ritornava a scrivere di Canova e del ritratto scultoreo di Henryk Lubomirski, sostenendo che lo scultore era riuscito a trasfondere nel marmo il calore e la morbidezza della carne propria di un adolescente e sottolineando il connubio tra bellezza e nudo adolescenziale:

A chi volesse troppo metafisicare sul bello, potrebbe forse parer cosa strana, che siasi cercato il bello nella macchina umana, prima ch'essa sia giunta alla perfezione, e quando le parti del corpo restano in certa maniera avviluppate, e non spiegano energicamente il carattere loro, somigliando alle frondi raggruppate di un fiore, prima che sia esso giunto al suo stato di maturità. Nonostante, lasciando a parte queste riflessioni, sappiamo, che i Greci cercavano il bello, e lo amarono con avidità in figure simili ${ }^{27}$.

Avendo ottenuto dalla principessa la massima libertà espressiva, Canova decise di rappresentare Henryk sotto le sembianze del dio Eros. La scultura, che si ispirava chiaramente alla statuaria antica in particolare all'Eros alato della collezione Farnese $^{28}$, afferiva a quel filone di nudo nel quale l'artista veneto aveva già dato alcune prove come il gruppo scultoreo di Orfeo e Icaro, realizzato nel 1779 e oggi custodito al Museo Correr di Venezia, e Apollo che si incorona,

25 D'Este, op. cit., p. 78.

26 G. Pavanello (2004), “Antonio D’Este: le memorie di Antonio Canova”, [in:] Simonetta La Barbera (a cura di), Gioacchino di Marzo e la critica d'arte nell'Ottocento in Italia, atti del convegno Palermo 2003, Bagheria, Officine Tipografiche Aiello e Provenzano, pp. 22-28.

27 "Memorie per le belle Arti" (1788), 4, p. 234.

28 B. Trojnar (2007), La statua di Henryk Lubomirski come Amore di Antonio Canova nel castello di Łańcut, [in:] Mario Guderzo (a c. di), Antonio Canova: il principe Henryk Lubomirski come Amore, Cinisello Balsamo (Milano), Silvana editoriale, p. 65. 
databile tra il 1781 e il 1782 e oggi esposto al Getty Museum di Los Angeles. Il risultato è una figura di adolescente nudo a grandezza naturale che impugna con la mano sinistra un arco ornato da fiori, mentre la faretra con le frecce è appesa con un nastro al tronco a cui sembra lievemente accostarsi il corpo, il cui peso del corpo grava sulla gamba destra, il braccio destro scende invece lungo il fianco. La testa dalla fluente capigliatura è rivolta di tre quarti sulla sua sinistra. Il viso presenta grandi occhi, un naso sottile e labbra semiaperte.

Dal Libro de' Conti risulta che Canova cominciava a modellare l'argilla probabilmente durante il mese di aprile, passava quindi al gesso ${ }^{29}$ nel giugno del 1786, realizzando un modello in scala naturale, che lo impegnava per soli diciannove giorni: il 2 luglio infatti il gesso era pronto e nel mese di ottobre il giovane aiutante di atelier Gaetano Cerotti ${ }^{30}$ passava a sbozzare il marmo, un'operazione meccanica che Canova lasciava nelle mani dei suoi collaboratori, impegnato com'era a ultimare il monumento scultoreo di Clemente XIV. Canova aveva perfezionato una tecnica destinata a far scuola dopo di lui, ma non del tutto nuova, perché già nota nel Rinascimento. Essa consisteva nell'uso di modelli di grandezza naturale in gesso contrassegnati da punti cioè chiodi in bronzo. Successivamente il modello in gesso e il blocco di marmo venivano posti sotto l'uno accanto all'altro sotto una cornice, detta da Canova "tellaro" 31 , da cui si partivano dei fili di piombo che, toccando i punti nel gesso e il marmo servivano per misurare le distanze e quindi per calcolare i volumi in maniera più rigorosa e scientifica ${ }^{32}$.

Inoltre, dal Libro de' Conti ${ }^{33}$ risulta che in quei giorni Canova acquistò per soli 20 scudi una statua antica dallo scultore e restauratore romano Carlo Albacini $^{34}$ (circa 1739-post 1807) che, a giudicare dal prezzo contenuto, doveva essere di ben poco valore ${ }^{35}$.

La statua venne infine collocata su un piedistallo cilindrico in marmo decorato con aquile e ghirlande d'alloro, sopra le quali poggia una farfalla, simbolo di Psiche, in riferimento al mito di Amore e Psiche. Per quanto concerne il piedistallo gli studiosi non sono concordi nel ritenerlo opera originale del Canova ${ }^{36}$.

29 Oggi a Possagno (Treviso), Gipsoteca (ala Lazzari), Amorino, Modello originale in gesso $(117 \times 53 \times 40 \mathrm{~cm}$, inv. 73$)$.

30 B. Trojnar, op. cit., pp. 64-65.

31 A. Canova, op. cit., p. 241.

32 www.museocanova.it/come-lavorava-antonio-canova/ [01/03/2020].

33 A. Canova, op. cit., p. 246.

34 M. Pepe (1960), Albacini, Carlo, in Dizionario Biografico degli Italiani, vol. 1, Roma, Istituto della Enciclopedia Italiana, p. 588.

35 H. Honour (1973), op. cit., p. 312; H. Honour (2007), op. cit., p. 29.

36 B. Trojnar, op. cit., p. 66. 
Nel 1790 la scultura fu inviata in Polonia e collocata al castello di Łańcut ${ }^{37}$, dove si trova ancora oggi circondata da quattro colonne ioniche di colore grigio a mo' di tempietto ${ }^{38}$.

Il piccolo principe del Canova ebbe subito una fama e risonanza internazionale, come provano le repliche marmoree dai tratti più idealizzati che derivano probabilmente dal gesso originale ${ }^{39}$. In particolare, quella richiesta dal colonello John Campbell (1755-1821), intorno al 1787 è custodita alla Anglesey Abbey a Cambridge, la seconda replica dell'Amorino per John La Touche (1772-1838) si trova alla National Gallery di Dublino e la terza copia completata nel 1795 per il principe russo Nicolaj Jussupov (1750-1831) è esposta all'Ermitage a San Pietroburgo ${ }^{40}$.

Il marmo di Łańcut è tornato in Italia nel luglio del 2007 nell'ambito delle celebrazioni a Possagno per i duecentocinquanta anni dalla nascita di Antonio Canova che coincidono anche con i duecentotrenta anni dalla nascita di Henryk Lubomirski ${ }^{41}$. In quell'occasione sono stati eseguiti calchi 3D che hanno permesso di restaurare il gesso originale posseduto dalla Gipsoteca e Museo Canova di Possagno, acefalo e privo di una spalla per gli effetti del bombardamento del novembre $1917^{42}$.

\section{Bibliografia}

\section{Fonti a stampa}

"Memorie per le belle Arti” (1788), 4, pp. 234-235.

Memorie di Antonio Canova scritte da Antonio D'Este e pubblicate per cura di Alessandro D'Este con note e documenti, Firenze, Le Monnier, 1864.

Canova, Antonio (2007). Scritti, Hugh Honour e Paolo Mariuz (a c. di), Roma, Salerno editrice. Winckelmann, Johann Joachim (1767). Dell'arte del disegno de' Greci e della bellezza, in Monumenti antichi inediti, vol. 1, Roma, [Pagliarini], 2 voll.

37 W. K. Wojtowicz (2007), Łańcut, il castello di Amore, [in:] Mario Guderzo (a c. di), Antonio Canova: il principe Henryk Lubomirski come Amore, Cinisello Balsamo (Milano), Silvana editoriale, pp. 55-61.

38 B. Trojnar, op. cit., p. 66.

39 E. Bassi (1973), "Larte del Canova nella cultura polacca", [in:] Vittore Branca (a c. di), Italia Venezia e Polonia tra Illuminismo e Romanticismo, Firenze, Olschki, p. 107; H. Honour (1973), op. cit., p. 315; H. Honour (2007), op. cit., p. 30.

40 H. Honour (1973), op. cit., p. 312; H. Honour (2007), op. cit., pp. 30-31.

41 B. Trojnar, op. cit., p. 69.

42 G. Delfini Filippi (2007), "L’Amorino di Possagno: storia di un modello in gesso di Antonio Canova e della sua conservazione, [in:] Mario Guderzo (a c. di), Antonio Canova: il principe Henryk Lubomirskicome Amore, Cinisello Balsamo (Milano), Silvana editoriale, p. 94; P. Mariuz (2007), Antonio Canova a Possagno: scultore, pittore, architetto, [in:] Mario Guderzo (a c. di), Antonio Canova: il principe Henryk Lubomirski come Amore, Cinisello Balsamo (Milano), Silvana editoriale, p. 90. 


\section{Studi}

Bassi, Elena (1973). "Larte del Canova nella cultura polacca”, [in:] Vittore Branca (a c. di), Italia Venezia e Polonia tra Illuminismo e Romanticismo, Firenze, Olschki, pp. 107-125.

Busiri Vici, Andrea (1988). "I turisti polacchi a Roma nella seconda metà del Settecento", Strenna dei Romanisti 44, pp. 73-90.

Cesareo, Antonello (2002). “Gavin Hamilton (1723-1798): a gentleman of probity, knowledge and real taste", Saggi e memorie di storia dell'arte 26, pp. 211-322.

Cunial, Giancarlo (2007). "Amore che legò per sempre Canova ai Polacchi”, [in:] Mario Guderzo (a c. di), Antonio Canova: il principe Henryk Lubomirski come Amore, Cinisello Balsamo (Milano), Silvana editoriale, pp. 43-53.

Delfini Filippi, Gabriella (2007). "L'Amorino di Possagno: storia di un modello in gesso di Antonio Canova e della sua conservazione, [in:] Mario Guderzo (a c. di), Antonio Canova: il principe Henryk Lubomirski come Amore, Cinisello Balsamo (Milano), Silvana editoriale, pp. 93-97.

Franchi, Saverio (1994-2002). Le impressioni sceniche. Dizionario bio-bibliografico degli editori e stampatori romani e laziali di testi drammatici e libretti musicali dal 1579 al 1800, Roma, Edizioni di storia e letteratura.

Honour, Hugh (1973). “Gli Amorini del Canova”, Arte illustrata 6, pp. 312-320.

Honour, Hugh (2007). Amorini del Canova, [in:] Mario Guderzo (a c. di), Antonio Canova: il principe Henryk Lubomirski come Amore, Cinisello Balsamo (Milano), Silvana editoriale, pp. 29-41.

Leone, Francesco (1999). "Giuseppe Angelini (1738-1811). Storia, committenze e rapporti con l'Europa nel culto della classicità', Ricerche di storia dell'arte 68, pp. 4-16.

Lorentz, Stanisław (1962). Relazioni artistiche fra l'Italia e la Polonia, Roma, Signorelli.

Marini, Giorgio (a c. di) (1988). Volpato Giovanni 1735-1803, Bassano del Grappa (Vicenza), Ghedina \& Tassotti.

Mariuz, Paolo (2007). Antonio Canova a Possagno: scultore, pittore, architetto, [in:] Mario Guderzo (a c. di), Antonio Canova: il principe Henryk Lubomirski come Amore, Cinisello Balsamo (Milano), Silvana editoriale, pp. 85-91.

Mazzocca, Fernando (2002). Giovanni Volpato mentore di Canova, [in:] Ferdinando Mazzocca, Enrico Colle (a c. di), Il neoclassicismo in Italia da Tiepolo a Canova, Milano, Skira, pp. 369-373.

Mazzocca, Fernando (2002). Antonio Canova, il "Fidia rinascente" tra antico e classico [in:] Ferdinando Mazzocca, Enrico Colle (a c. di), Il neoclassicismo in Italia da Tiepolo a Canova, Milano, Skira, pp. 375-404.

Munõz, Antonio (1924/1925). "Il periodo veneziano di Antonio Canova e il suo primo maestro", Bollettino d'arte del Ministero della Pubblica Istruzione 18, pp. 103-128.

Pavanello, Giuseppe (2004). “Antonio D’Este: le memorie di Antonio Canova”, [in:] Simonetta La Barbera (a cura di), Gioacchino di Marzo e la critica d'arte nell'Ottocento in Italia, atti del convegno Palermo 2003, Bagheria, Officine Tipografiche Aiello e Provenzano, pp. 22-28.

Pavanello, Giuseppe (2014). "Su una prima idea di Canova per il monumento funerario di Clemente XIV”, [in:] Vincenzo Cazzato, Sebastiano Roberto e Mario Bevilacqua (a c. di) La Festa delle Arti. Scritti in onore di Marcello Fagiolo per i cinquant'anni di studi, Roma, Gangemi, vol. 2, pp. 772-775. 
Pepe, Mario (1960). Albacini, Carlo, in Dizionario Biografico degli Italiani, vol. 1, Roma, Istituto della Enciclopedia Italiana, p. 588.

Rigon, Ferdinando (1982), "Disegni del Canova del Museo di Bassano”, [in:] Giulio Carlo Argan, Barbieri Franco, Ferdinando Rigon (a c. di), Disegni del Canova del Museo di Bassano, Milano, Electa, pp. 14-27.

Rita, Andreina (1991). De Rossi, Giovanni Gherardo, [in:] Dizionario Biografico degli Italiani, vol. 39, Roma, Istituto della Enciclopedia Italiana, pp. 214-218.

Stefani, Ottorino (1990). I rilievi del Canova. Una nuova concezione del tempo e dello spazio, Milano, Electa.

Trojnar, Barbara (2007). La statua di Henryk Lubomirski come Amore di Antonio Canova nel castello di Łańcut, [in:] Mario Guderzo (a c. di), Antonio Canova: il principe Henryk Lubomirski come Amore, Cinisello Balsamo (Milano), Silvana editoriale, pp. 63-83.

Wojtowicz, Wit K. (2007). Łańcut, il castello di Amore, [in:] Mario Guderzo (a c. di), Antonio Canova: il principe Henryk Lubomirski come Amore, Cinisello Balsamo (Milano), Silvana editoriale, pp. 55-61.

Zanella, Andrea (2000). "Il monumento funerario papale da Bernini a Canova", [in:] Giuseppe Pavanello (a c. di) Antonio Canova e il suo ambiente artistico fra Venezia, Roma e Parigi, Venezia, Istituto Veneto di Scienze, Lettere ed arti, pp. 269-281.

\section{Sitografia}

museocanova.it/come-lavorava-antonio-canova/ [01/03/2020]. 